Check for updates

Cite this: RSC Adv., 2017, 7, 33043

\title{
Highly sensitive determination of non-steroidal anti-inflammatory drug nimesulide using electrochemically reduced graphene oxide nanoribbons
}

\author{
Mani Govindasamy, (D) a Veerappan Mani, (DD ${ }^{\text {ab }}$ Shen-Ming Chen, (DD *a \\ Thandavarayan Maiyalagan, ${ }^{c}$ S. Selvaraj, ${ }^{d}$ Tse-Wei Chen, ${ }^{a}$ Shih-Yi Lee ${ }^{e}$ \\ and Wen-Han Chang ${ }^{\text {fgh }}$
}

The excess use of nimesulide (NIM) causes acute side effects to gastrointestinal, central nervous and genitourinary systems and hence its rapid, sensitive determination is highly important. We describe a robust electrochemical sensor based on electrochemically reduced graphene oxide nanoribbons (ERGONRs) modified on a screen-printed carbon electrode (SPCE) for detecting NIM in pharmaceutical formulations and biological medium. Compared with parental multiwalled carbon nanotubes (MWCNTs), ER-GONRs possess rich edge defects, abundant functional groups, high area-normalized edge-plane structures and chemically active sites and hence they can be a superior electrocatalyst and signal amplifier for electroanalytical applications. ER-GONRs/SPCE exhibited excellent sensing performance towards NIM. The linear range was $1.0 \times 10^{-8}$ to $1.50 \times 10^{-3} \mathrm{M}$ and the detection limit was 3.50 $( \pm 1.57) \mathrm{nM}$. In addition, the ER-GONRs/SPCE showed excellent real-time sensing applications in NIM tablet and human urine samples, which could find potential applicability in drug and clinical analysis. The combined advantages of SPCE technology and ER-GONRs make this method a robust, low-cost, reproducible, sensitive and easy-to-use sensor.

Received 8th March 2017 Accepted 27th May 2017 DOI: $10.1039 / \mathrm{c} 7 \mathrm{ra02844j}$ rsc.li/rsc-advances (abdominal discomfort, heartburn, abdominal cramps, nausea, vomiting and diarrhea), central nervous (headache, dizziness and drowsiness) and genitourinary (blood in urine, decrease in urination and kidney failure) systems. ${ }^{3}$ Therefore, sensitive determination of NIM at trace levels is highly important. ${ }^{1,4}$ Generally, chromatographic methods ${ }^{5}$ coupled with spectrometric read-out are the major methods for NIM detection due to their high level of selectivity and sensitivity; however these methods are laborious, expensive, bulky, and require long assay times and pre-concentration steps. ${ }^{6}$ On the other hand, electroanalytical methods are simple, cheaper, portable, easy-touse, miniaturizable, consume less power, direct digital signal and applicable in point-of-care use. ${ }^{7,8}$ Unmodified electrodes are encountering surface fouling issues, poor reproducibility and low sensitivity. ${ }^{9,10}$ Therefore, chemical modifiers have been developed for detecting NIM; ${ }^{\mathbf{4}, 11,12}$ however, the sensitivities of these modified electrodes were not satisfactory for real-time applications. Carbon based nanomaterials have enormous popularity in electroanalytical applications owing to their large surface area, high electrical conductivity and good mechanical and thermal stability. Zhang et al., achieved seven times increase in response current for the NIM detection at multiwalled carbon nanotubes (MWCNTs) film modified glassy carbon electrode (GCE) compared with unmodified electrode. ${ }^{13}$ \footnotetext{
Mechatronic Engineering, National Taipei University of Technology, Taiwan

${ }^{h}$ Graduate Institute of Injury Prevention and Control, Taipei Medical University, Department of Medicine, Taiwan

Department of Chemical Engineering and Biotechnology, National Taipei University of net; Fax: +886 2270 25238; Tel: +886227017147

University of Technology, Taipei, Taiwan, Republic of China

'SRM Research Institute, Department of Chemistry, SRM University, Kattankulathur-

${ }^{d}$ Bishop Heber College (Autonomous), Tiruchirappalli-620017, Tamil Nadu, India

${ }^{e}$ Division of Pulmonary and Critical Care Medicine, MacKay Memorial Hospital, MacKay Medicine, Nursing and Management College, Taiwan

${ }^{f}$ MacKay Memorial College Department of Cardiology, MacKay Memorial Hospital, Taiwan

${ }^{g}$ Department of Emergency Medicine, MacKay Memorial Hospital, Institute of
} 
Similarly, Wang et al., observed improved sensitivity for NIM using cysteic acid/MWCNTs film modified electrode. ${ }^{\mathbf{1 4}}$ Recent studies revealed that graphene nanoribbons (GNRs) or reduced graphene oxide nanoribbons (R-GONRs), narrow strips of graphene nanosheets have the highest edge density and edge defects over all the other carbon based nanomaterials. ${ }^{15}$ Moreover, GNRs have higher area-normalized edge-plane structures and chemically active sites than that of graphene, MWCNTs and graphite; as a result, GNRs can be superior electrode material in electroanalytical chemistry. Therefore, recently many efforts are focusing on the preparation of GNRs or R-GONRs based nanomaterials for electroanalytical applications. ${ }^{\mathbf{1 6 - 2 4}}$

Herein, we aimed to describe electrochemically reduced graphene oxide nanoribbons (ER-GONRs) film modified screenprinted carbon electrode (SPCE) for determination of NIM (Scheme 1). The graphene oxide nanoribbons (GONRs), an oxygenated derivative was prepared via longitudinal unzipping of MWCNTs. The oxygen functionalities located on the basal and edge planes of GONRs allow it to form aqueous dispersion and provide sites for functionalization. The ER-GONRs film was prepared through drop casting GONRs with subsequent electrochemical reduction. Remarkably, many of the previous reports studied the electrochemical oxidation of NIM, which usually occurs at higher overpotential region and encounters interference from biological interferents. In this work, we have studied the reduction of NIM and successfully demonstrated the working principle in real samples without interference. The main objective of this work is to develop highly sensitive chemically modified electrode, ER-GONRs/SPCE for the detection of NIM (Scheme 1). To the best of our knowledge, this is the first report on GNRs based materials for the reduction of NIM. Interestingly, the detection limit achieved by the fabricated ERGONRs/SPCE is $3.50( \pm 1.57) \mathrm{nM}$ which surpassed the detect limits of many previously reported NIM sensor. In comparison with classical electrodes, the SPCEs have improved sensitivity and selectivity for the sensing applications in addition with a series of advantages such as simplicity, low-cost, commercial availability, reproducibility and repeatability.

\section{Experimental}

\subsection{Chemicals and apparatus}

MWCNTs (bundled > 95\%), and all other reagents including solvents were purchased from Sigma-Aldrich and used as received. Electrochemical studies were performed in a conventional three electrode cell using modified SPCE (Bioanalytical Systems, Inc., USA) as a working electrode (area 0.3 $\mathrm{cm}^{2}$ ), saturated $\mathrm{Ag} \mid \mathrm{AgCl}$ (saturated $\mathrm{KCl}$ ) as a reference electrode and Pt wire as a counter electrode. The SPCEs were purchased from Zensor R\&D Co., Ltd., Taiwan.

All the electrochemical measurements were carried out using CHI 1205A electrochemical work station ( $\mathrm{CH}$ Instruments, Inc., U.S.A) at ambient temperature. Prior to each electrochemical experiment, the electrolyte solutions were deoxygenated with pre-purified nitrogen for $15 \mathrm{~min}$ unless otherwise specified. The supporting electrolyte used for the electrochemical studies was $0.1 \mathrm{M}$ phosphate buffer $(\mathrm{PB})(\mathrm{pH}$ 7.4) prepared from sodium dihydrogen phosphate and disodium hydrogen phosphate. 0.1 M acetate buffer (for pH 3 and 5), $0.1 \mathrm{M}$ phosphate buffer (for $\mathrm{pH} 7$ ) and 0.1 M Tris-buffered saline (for $\mathrm{pH}$ 9) were prepared and used for different $\mathrm{pH}$ studies. Surface morphological studies were carried out using transmission electron microscope (TEM) (H-7600, Hitachi, Japan). Energy-dispersive X-ray (EDX) spectra were performed using Horiba Emax x-act (sensor $+24 \mathrm{~V}=16 \mathrm{~W}$, resolution at $5.9 \mathrm{keV}$ ). EIM6ex Zahner (Kronach, Germany) was used for electrochemical impedance spectroscopy (EIS) studies. X-ray diffraction (XRD) diffraction studies were carried out using XPERTPRO (PANalytical B.V., The Netherlands) diffractometer (Cu Ka radiation, $k=1.54 \AA)$. Raman spectra were acquired using Micro-Raman spectrometer (RENISHAW in via system, U.K) by a $514.4 \mathrm{~nm} \mathrm{He} / \mathrm{Ne}$ laser. FTIR spectra were carried out using a Perkin-Elmer IR spectrometer.

\subsection{Preparation of ER-GONRs}

ER-GONRs were prepared by reported procedure with little modification. ${ }^{25} 200 \mathrm{mg}$ of MWCNTs were added to $80 \mathrm{~mL} \mathrm{H}_{2} \mathrm{SO}_{4}$ and stirred for $1 \mathrm{~h}$. Then, $6 \mathrm{~mL}$ of $\mathrm{H}_{3} \mathrm{PO}_{4}$ was added and the whole solution was continuously stirred for $30 \mathrm{~min}$. Subsequently, the whole mixture was heated at $70{ }^{\circ} \mathrm{C}$ for $2 \mathrm{~h}$ and then cooled to room temperature. Next, the reaction mixture was poured onto $150 \mathrm{~mL}$ of ice containing $10 \mathrm{~mL}$ of $30 \% \mathrm{H}_{2} \mathrm{O}_{2}$. Brown colored sediment was obtained which was filtered and washed with $100 \mathrm{~mL}$ water. Next, it was washed with $3 \times \mathrm{HCl}(20 \mathrm{vol} \%, 50$ $\mathrm{mL}$ each), and $2 \times$ ethanol (50 mL each). Finally, the purified GONRs were vacuum dried at $60{ }^{\circ} \mathrm{C}$ for overnight. $1 \mathrm{mg} \mathrm{mL}{ }^{-1}$ of GONRs was prepared in water via ultrasonication (30 min) and this dispersion is highly stable in water for more than a month.

Among all the reported preparation methods, longitudinal unzipping through acid oxidation of MWCNTs is the suitable
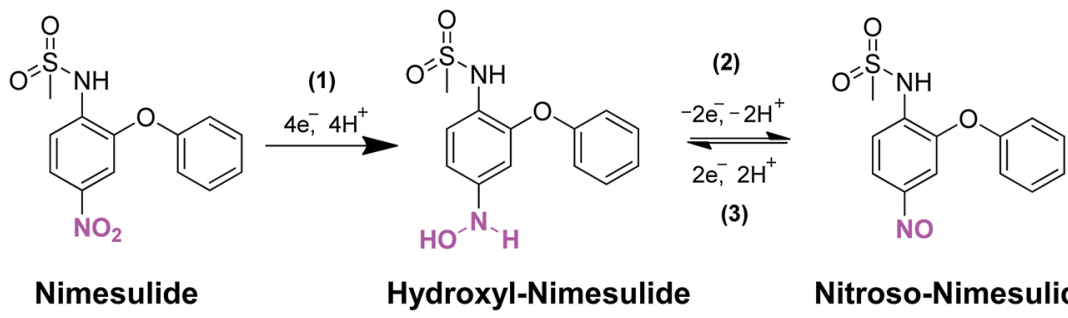

Nitroso-Nimesulide 
method for mass production of ER-GONRs. Interestingly, this method of ER-GONRs production creates plethora of structural defects on the ribbons that are actually beneficial for electrocatalytic sensing applications. The functional groups located at the edges of ER-GONRs able to facilitate the adsorption of analyte by $\pi-\pi$ stacking, electrostatic, hydrogen bonding, and covalent interactions. ${ }^{26}$ All assays were performed in triplicate, and the results reported are the average of at least three experiments.

\subsection{Preparation of ER-GONRs film modified SPCE}

First, the surface of SPCE was pre-cleaned by cycling between $-1.0 \mathrm{~V}$ and $1.2 \mathrm{~V}$, in $0.1 \mathrm{M} \mathrm{PB}(\mathrm{pH} 7.0){ }^{27}$ Then, $6 \mu \mathrm{L}$ of GONRs (optimized amount) was drop casted on the pre-cleaned SPCE surface and dried. Next, the ER-GONRs/SPCE was transferred to an electrochemical cell containing deaerated $0.1 \mathrm{M} \mathrm{PB}(\mathrm{pH}$ 5.0). 20 consecutive cycles were performed in the potential range of 0 to $-1.50 \mathrm{~V}$ and the scan rate was held at $0.1 \mathrm{~V} \mathrm{~s}^{-1}$. Then, the ERGONRs/SPCE was removed from the cell and gently washed with water and dried at ambient conditions before employed in sensor experiments. The electrochemical reduction pattern was in accordance with our previous reports which indicating the reduction of oxygen functionalities of graphene oxide. ${ }^{28}$ MWCNTs/SPCE and ER-GONRs/SPCE were prepared separately simply by drop casting the respective materials on the pre-cleaned SPCE and these electrodes were used for control experiments.

\subsection{Preparation of real samples}

Nimesulide tablet was purchased from local pharmacy in Tamil Nadu, India and grinded with mortar. The powdered NIM tablet was dissolved in $0.1 \mathrm{M} \mathrm{PB}(\mathrm{pH} 7)$ to get the corresponding standard NIM stock solution and used for the amperometry studies. Drug free urine samples collected from healthy volunteer was diluted to 100 times by adding $0.1 \mathrm{M} \mathrm{PB} \mathrm{(pH} \mathrm{7).}$ Informed consent was obtained from all subjects for urine collection. Known amounts of NIM sample was spiked into the diluted urine sample and amperometry experiments were performed. All assays were performed in triplicate, and the results reported are the average of at least three experiments.

\section{Results and discussions}

\subsection{Surface morphological and elemental characterizations}

The TEM image of MWCNTs (Fig. 1A) displays the typical tubular nanostructure and the corresponding EDX spectrum (Fig. 1B) revealed the presences of expected elements, carbon (C) and oxygen (O) with weight\% of 96.27 and 3.73, while atomic\% of 96.91 and 3.09, respectively. The TEM image of GONRs displays the thin sheets of nanoribbons with smooth edges, while the lengths of the ribbons were about $>5 \mu \mathrm{m}$, and the widths ranging from $80-300 \mathrm{~nm}$, those were consistent with the previous reports ${ }^{25}$ (Fig. 1C). The EDX analysis of GONRs (Fig. 1D) confirmed the presences of $\mathrm{C}$ and $\mathrm{O}$ with weight\% of 60.37 and 39.63 , while atomic\% of 78.61 and 21.39 , respectively. The TEM image of ER-GONRs also displayed similar morphology of nanoribbons (Fig. 1E), while the corresponding
EDX spectrum (Fig. 1F) featured with $\mathrm{C}$ and $\mathrm{O}$ with weight\% of 93.48 and 6.52 , while atomic\% of 95.25 and 4.75 , respectively. As expected, the amount of oxygen is significantly decreased from GONRs to ER-GONRs. As established in graphene literature, the oxygen functionalities resided on the edges and planes of the sheets are partially removed during reduction, which led to the considerable reduction in the amount of oxygen. ${ }^{28}$

\subsection{XRD, Raman, FT-IR and impedance characterizations}

Raman spectroscopy is used to obtain information about the degree of disorder in graphene materials. The general density of defects in the $\mathrm{sp}^{2}$ lattice can be elucidated based on the intensity ratio of $\mathrm{D}$ band to $\mathrm{G}$ band $\left(I_{\mathrm{D}} / I_{\mathrm{G}}\right)$ and location of the bands. ${ }^{29}$ The Raman spectra of MWCNTs (a), GONRs (b) and ER-GONRs (c) were shown in Fig. 2A. The spectra displayed three major bands; D, G and $2 \mathrm{D}$ bands which are manifested to the breathing mode of $k$-point phonons of $\mathrm{A}_{1 \mathrm{~g}}$ symmetry (defect related), the $\mathrm{E}_{2 \mathrm{~g}}$ planar configuration of $\mathrm{sp}^{2}$ bonded carbon (denotes $\mathrm{sp}^{2}$ lattice) and second order overtone of the $\mathrm{D}$ band (represents thickness of the sheets). The D, G and 2D bands for MCWNTs were observed at 1354, 1574 and $2705 \mathrm{~cm}^{-1}$. After MWCNTs converted into GONRs, the $I_{\mathrm{D}} / I_{\mathrm{G}}$ intensity ratio increased from 0.75 to 0.94 and the $2 \mathrm{D}$ band is almost disappeared. As evidently, the oxidation of MWCNTs to GONRs and generation of numerous edge sites on the sheets are led to decrease in the average size of in-plane $\mathrm{sp}^{2}$ domain next, the spectrum of GONRs and ER-GONRs were compared. Again, the $I_{\mathrm{D}} / I_{\mathrm{G}}$ intensity ratio increased from 0.94 (GONRs) to 1.01 (ERGONRs) in addition with the reappearance of $2 \mathrm{D}$ band and the result clearly revealing the formation of graphitic domains as a result of electrochemical reduction and the overall Raman result is consistent with previous reports. ${ }^{30}$

Fig. 2B displays the XRD patterns of MWCNTs (a), GONRs (b) and ER-GONRs (c). The diffraction pattern of MWCNTs featured with the characteristic peak at $2 \theta$ angle of $26.5^{\circ}$, while it was shifted to $10.5^{\circ}$ in the spectrum of GONRs, which is due to the incorporation of oxygen functionalities on the surface of nanoribbons. However, this diffraction peak shifted back to $26.5^{\circ}$ in the spectrum of ER-GONRs indicating the removal of oxygen functionalities of GONRs via electrochemical reduction and restoration of electrical conductivity. Next, the FTIR analysis is carried out in order elucidate information regarding functional groups variations in MWCNTs (a), GONRs (b) and ER-GONRs (c) (Fig. 2C). There are two major vibrational modes, at 3690 and $1530 \mathrm{~cm}^{-1}$ which were arose due to the vibrations of adsorbed water and $\mathrm{C}=\mathrm{C}$. The spectrum of GONRs exhibited well-defined characteristic peaks, $\nu(\mathrm{C}-\mathrm{O}-\mathrm{C})=1065, \nu(\mathrm{C}=\mathrm{C})=$ 1545, $\nu(\mathrm{C}=\mathrm{O})=1740$ and $\nu(-\mathrm{OH})=3430 \mathrm{~cm}^{-1}$ which are manifested to the stretching vibrations of representative oxygen functionalities residing on the ribbons. On the other hand, the intensities of these peaks were considerably decreased or even disappeared in the spectrum of ER-GONRs, which confirms the removal of oxygen functionalities that restore the conductivity.

SPCE surface modification with nanomaterials will change the double layer capacitance and interfacial electron transfer resistance of the electrode. Fig. 2D displays the EIS 

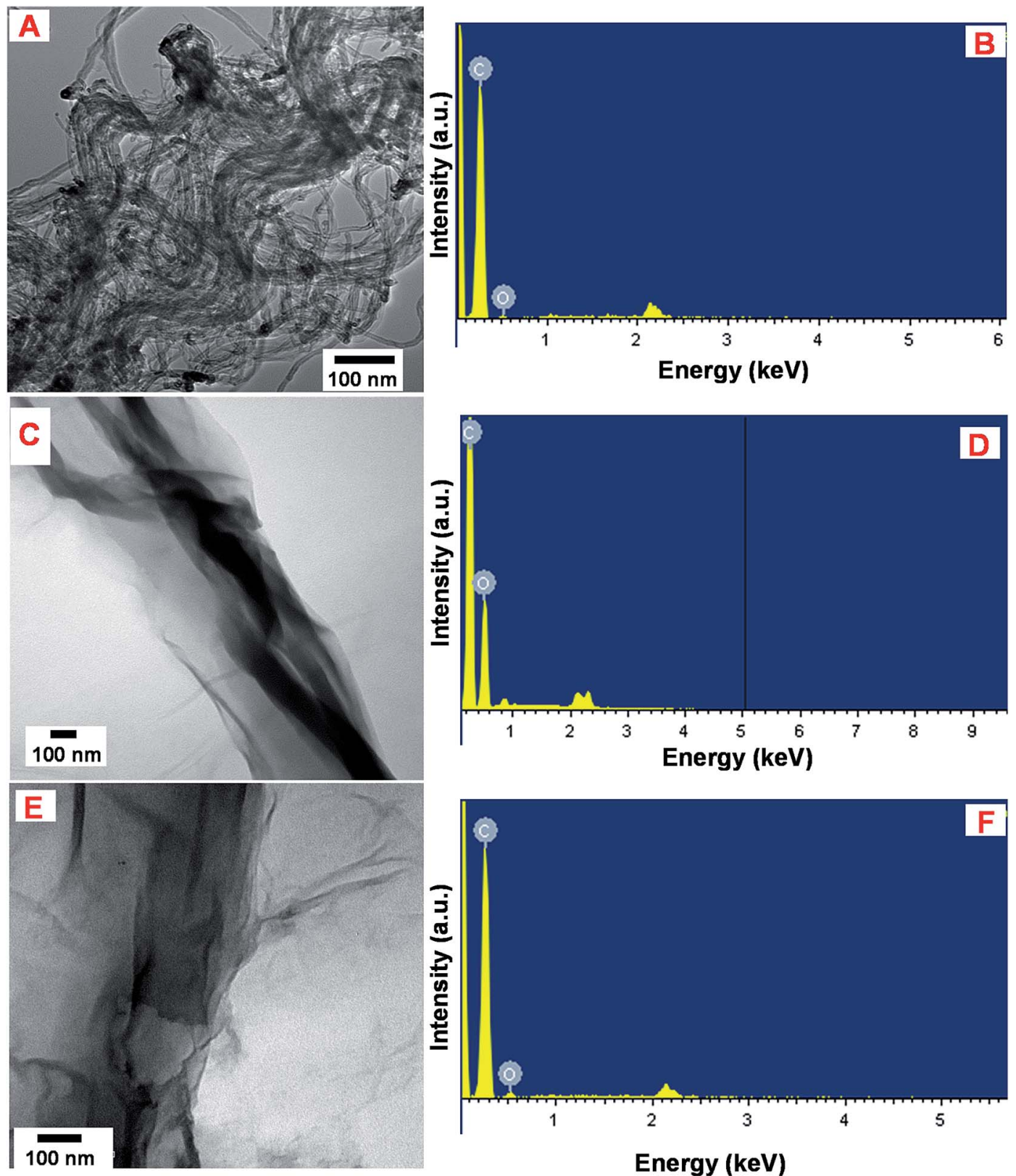

Fig. 1 TEM images of MWCNTs (A), GONRs (C) and ER-GONRs (E). EDX spectra of MWCNTs (B), GONRs (D) and ER-GONRs (F).

measurements of the modified electrodes represented as Nyquist plots; MWCNTs/SPCE (a), GONRs/SPCE (b) and ERGONRs/SPCE (c). Randles equivalent circuit model has been used to fit the experimental data (inset to Fig. 2D), in which, $R_{\mathrm{S}}$, $R_{\mathrm{ct}}, C_{\mathrm{dl}}$ and $Z_{\mathrm{w}}$ were representing electrolyte resistance, charge transfer resistance, double layer capacitance and Warburg impedance, respectively. The semicircle appeared in the Nyquist plot indicates the parallel combination of $R_{\mathrm{ct}}$ and $C_{\mathrm{dl}}$ at the electrode surface resulting from electrode impedance, while linear portion represents the diffusion limited process. It can be seen from the figure that the diameter of semicircles $\left(R_{\mathrm{ct}}\right)$ obtained for various electrodes are different; GONRs/SPCE > ERGONRs/SPCE > MWCNTs/SPCE. The $R_{\text {ct }}$ of GONRs/SPCE was higher than the MWCNTs/SPCE, because the surface of GONRs contains several oxygen functionalities which make this electrode poorly conductive. In other words, the presence of $\mathrm{sp}^{3}$ carbon atoms and disrupted delocalized $\mathrm{sp}^{2}$ network rendered the GONRs electrically insulating material. However, the $R_{\mathrm{ct}}$ was considerably lowered after it was reduced to ER-GONRs/SPCE since much of the oxygen functionalities were removed and $\mathrm{sp}^{2}$ network was partially restored.

\subsection{Electrocatalysis of nimesulide}

The electrocatalytic ability of ER-GONRs/SPCE towards reduction of NIM was investigated by cyclic voltammetry. The cyclic voltammograms are recorded at various electrodes in $\mathrm{PB}(\mathrm{pH} 7)$ containing $2 \mu \mathrm{M}$ NIM over the potential range of $+0.40 \mathrm{~V}$ to 

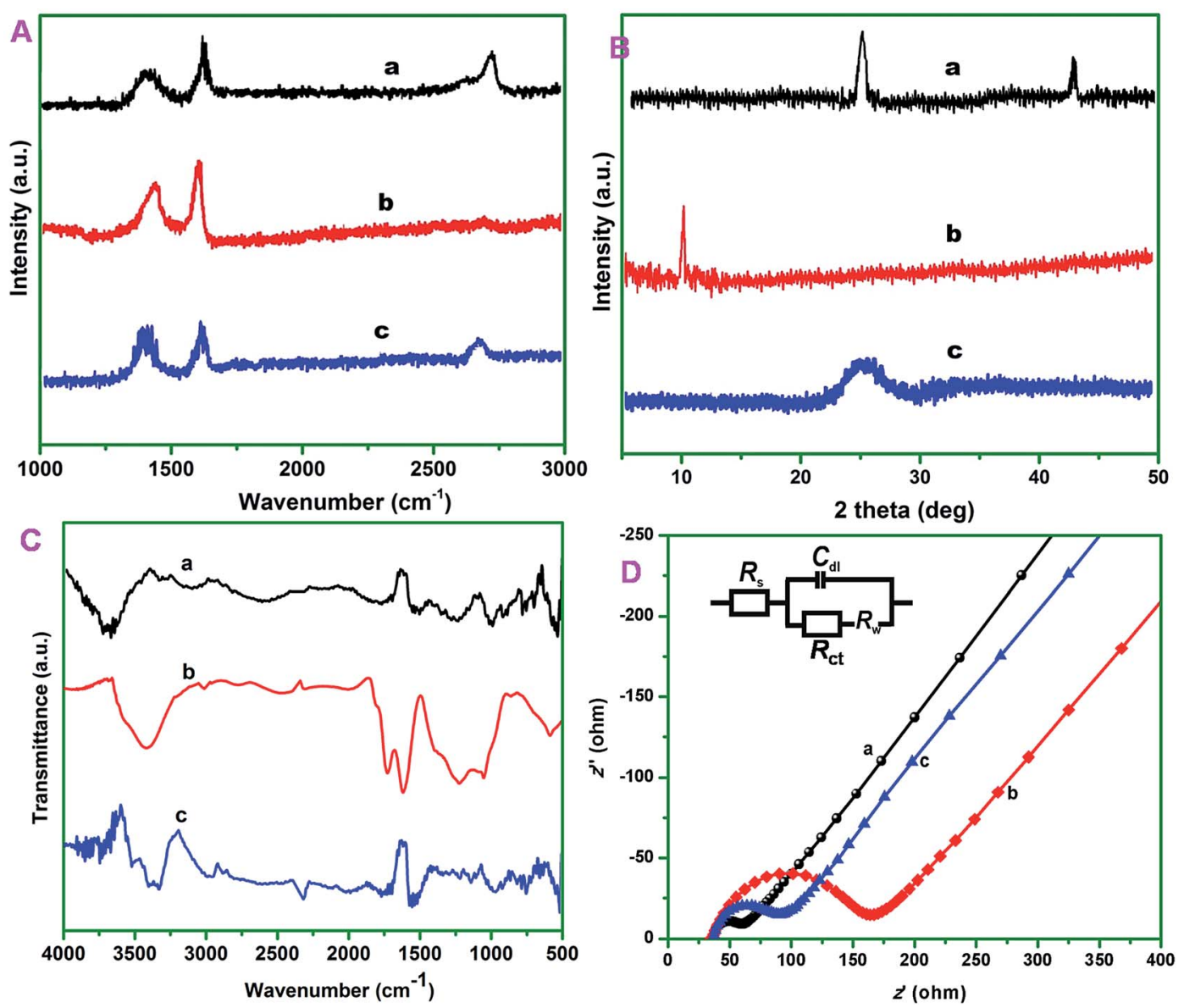

Fig. 2 (A) Raman, (B) XRD, (C) FT-IR and (D) EIS characterizations of MWCNTs (a), GONRs (b) and ER-GONRs (c). EIS spectra of MWCNTs (a), GONRs (b), and ER-GONRs (c) films modified SPCEs (D) in $0.1 \mathrm{M} \mathrm{KCl}$ containing Fe(CN) ${ }_{6}{ }^{3-14-}$. Inset: Randles equivalent circuit model; $R_{\mathrm{s}}, R_{\mathrm{ct}}, C_{\mathrm{dl}}$ and $Z_{w}$ are electrolyte resistance, charge transfer resistance, double layer capacitance and Warburg impedance, respectively.

$-0.80 \mathrm{~V}$ (Fig. 3A). The inset to Fig. 3A presents two consecutive voltammograms of ER-GONRs/SPCE in presence of $2 \mu \mathrm{M}$ NIM. In the forward scan, a cathodic peak, $I_{\mathrm{pc}}$ (peak $\mathrm{a}^{\prime}$ ) featured at $-0.60 \mathrm{~V}(v s$. $\mathrm{Ag} / \mathrm{AgCl}$ ) is corresponding to irreversible reduction of nitro group to hydroxyl amine. During the reverse scan and second cycle, a reversible redox peaks $\left(b^{\prime}\right.$ and $\left.c^{\prime}\right)$ are observed, which are identified as redox reactions of hydroxyl amine to nitroso. Thus, the electrocatalytic reduction mechanism of NIM follows typical nitrite reduction pathway as given in Scheme 1. At bare SPCE, feeble anodic peaks are observed for NIM. GONRs and MWCNTs films modified electrodes have shown sharp reduction peaks; however with small current signals (Fig. 3A). Remarkably, ER-GONRs/SPCE exhibits a tremendous increase in sensitivity with $I_{\mathrm{pa}}$ of $39.3 \mu \mathrm{A}$ which is 3.5 and 4.8 times higher than that of MWCNTs/SPCE and bare SPCE, respectively. This result reveals the high sensitivity of the composite film modified electrode towards NIM reduction. The significantly improved sensor performance of MWCNTs over ER-GONRs can be correlated to the higher area-normalized edge-plane structures, rich edge chemistry, abundant functionalities and chemically active sites of ER-GONRs. Fig. 3B displays the voltammograms obtained at ER-GONRs/SPCE towards different concentrations of NIM. The reduction peak current linearly increases with increase in the concentration of NIM and the plot between reduction peak current and concentration of NIM displays good linearity (inset to Fig. 3B).

\subsection{Effect of scan rate}

The effect of scan rate $(\nu)$ on the reduction of NIM has been studied by voltammetry in the potential range, $+0.40 \mathrm{~V}$ to $-0.80 \mathrm{~V}$ (Fig. 4A). The value of $I_{\mathrm{pc}}$ increases as the scan rate increases and the peak potential is negatively shifted. The inset of Fig. $4 \mathrm{~A}$ shows the plot of $\log \nu v s$. $\log I_{\mathrm{pa}}$. The dependence of $I_{\mathrm{pa}}$ on the square root scan rate can be represented as $I_{\mathrm{pa}}=263.16 \log \nu-$ $33.45, R^{2}=0.978$. Where, $I_{\mathrm{pa}}$ and $\nu$ are measured in $\mu \mathrm{A}$ and $\mathrm{V} \mathrm{s}^{-1}$ respectively. As evident from the plot, the square root of scan rate has linear dependence with $I_{\mathrm{pc}}$, a behaviour expected for diffusion controlled electrocatalytic process. The effect of scan rate on the $I_{\mathrm{pc}}$ of NIM oxidation at room temperature can be described by Randles-Sevcik equation as given in eqn (1),

$$
I_{\mathrm{p}}=2.99 \times 10^{5} n\left[(1-\alpha) n_{\alpha}\right]^{1 / 2} A C_{\mathrm{b}} D^{1 / 2} \nu^{1 / 2}
$$

here, $I_{\mathrm{p}}$ is the peak current, $n$ is the number of electrons, $\alpha$ is the electron transfer coefficient, $n_{\alpha}$ is the number of electrons involved in the rate-determining step, $A$ is the area of the 

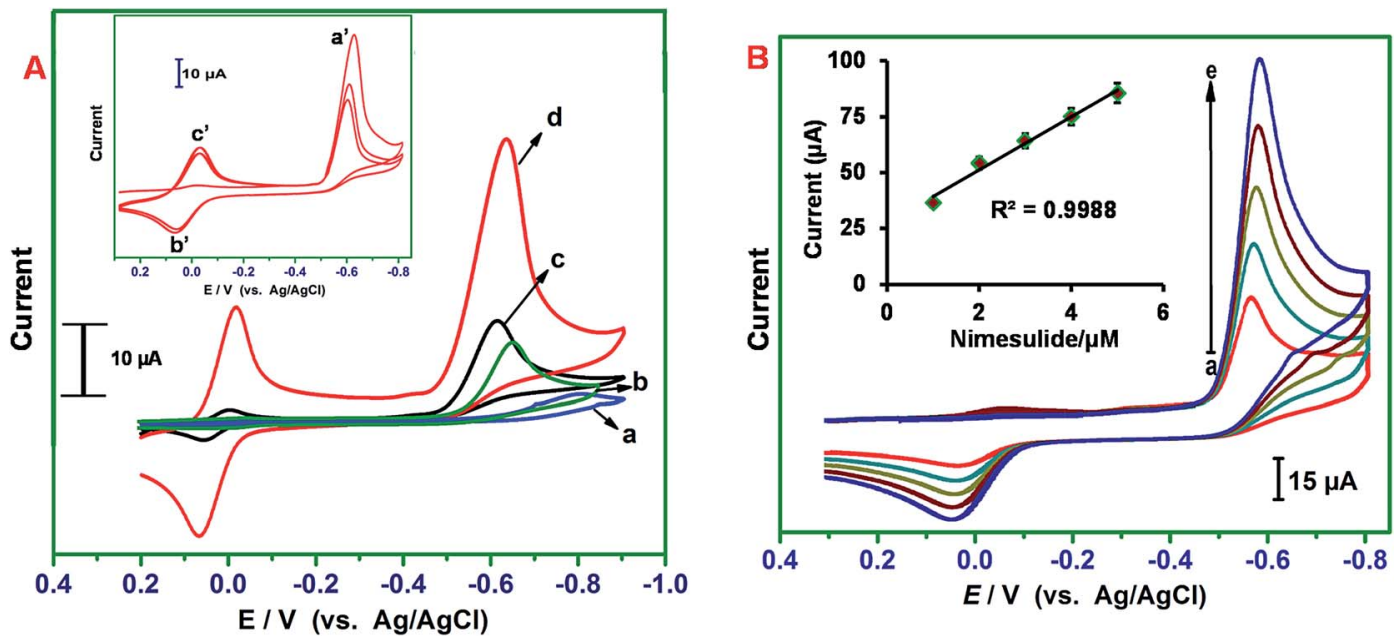

Fig. 3 (A) Cyclic voltammograms obtained at bare SPCE (a), GONRs/SPCE (b), MWCNTs/SPCE (c) and ER-GONRs/SPCE (d) in 0.1 M PB (pH 7.0) containing $2 \mu \mathrm{M}$ NIM. Inset: 3 continuous voltammograms at ER-GONRs/SPCE in $2 \mu \mathrm{M}$ NIM. (B) Cyclic voltammograms obtained at ER-GONRs/ SPCE in PB ( $\mathrm{pH} 7.0)$ containing nimesulide $(a=1 \mu \mathrm{M}, b=2 \mu \mathrm{M}, \mathrm{c}=3 \mu \mathrm{M}, d=4 \mu \mathrm{M}$ and $e=5 \mu \mathrm{M})$. Inset: [nimesulide] ( $\mu \mathrm{M})$ vs. reduction peak current $(\mu \mathrm{A})$. Scan rate $=50 \mathrm{mV} \mathrm{s}^{-1}$.
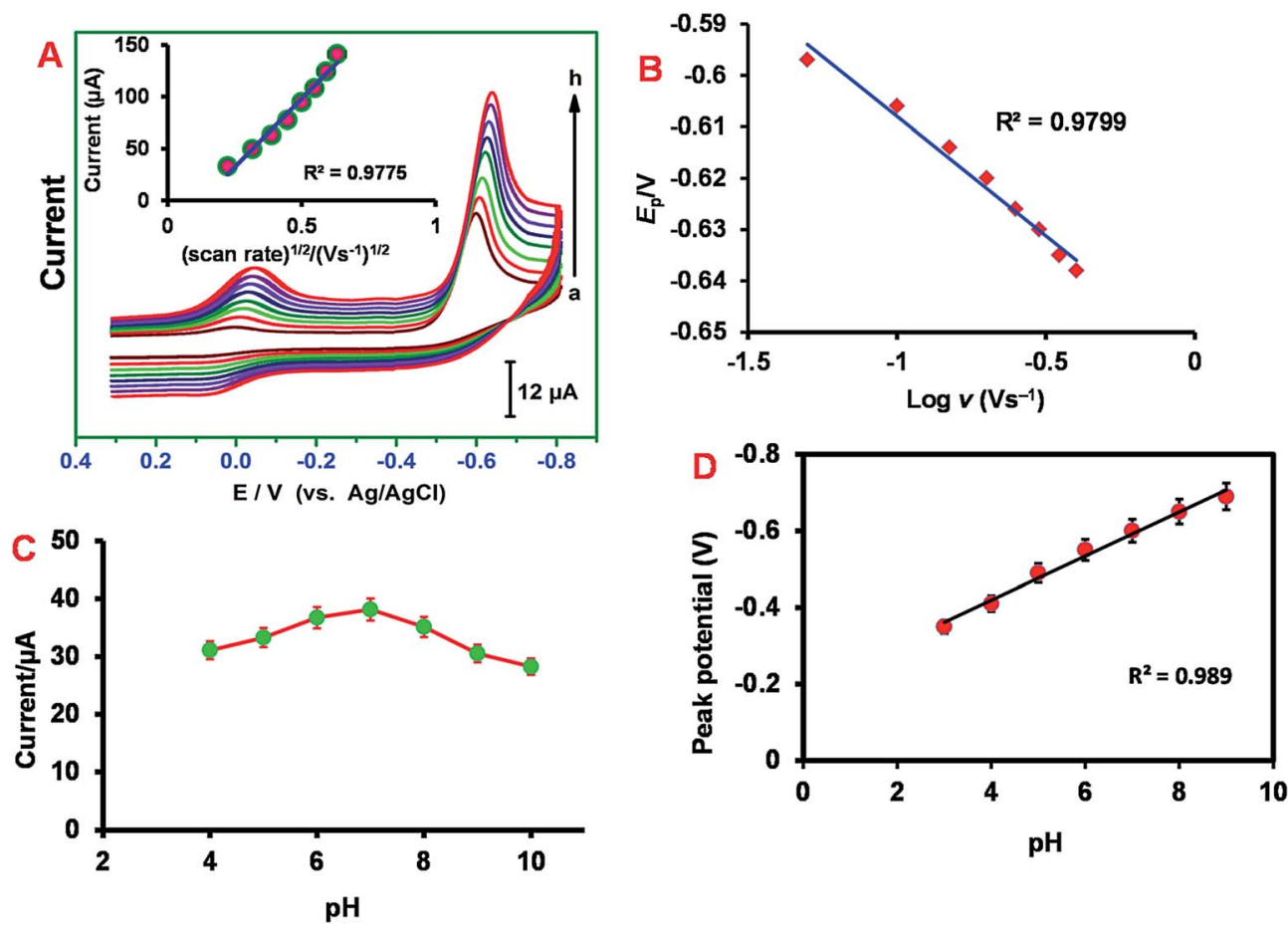

Fig. 4 (A) Cyclic voltammograms obtained at ER-GONRs/SPCE in PB (pH 7.0) containing $2 \mu \mathrm{M}$ NIM at different scan rates $(a=0.05, b=0.1, c=$ $0.15, d=0.20, d=0.25, f=0.30, g=0.35$ and $h=0.40)$. Inset: plot of (scan rate $)^{1 / 2}\left(V^{-1}\right)^{1 / 2} v s$. current $(\mu \mathrm{A})$. (B) Tafel plot: $E_{\mathrm{p}} v s$. log $\nu\left(V s^{-1}\right)$. (C) Plot between peak current $(\mu \mathrm{A})$ vs. $\mathrm{pH}$ and $(\mathrm{D})$ plot between peak potential $(\mathrm{V}) \mathrm{vs}$. $\mathrm{pH}$. Cyclic voltammograms are carried out in $\mathrm{PB}(\mathrm{pH} 7.0$ ) containing $2 \mu \mathrm{M}$ NIM.

electrode, $C_{\mathrm{b}}$ is the concentration of NIM, $D$ is the diffusion coefficient and $\nu$ is the scan rate.

Also, the peak potential $\left(E_{\mathrm{p}}\right)$ shifts to higher negative potentials with the increase in scan rate (Fig. 4B). A plot of $E_{\mathrm{p}} v s$. $\log \nu$ displays a linear relationship which implies that NIM electrocatalytic reduction process is chemically irreversible. The regression equation can be expressed as, $E_{\mathrm{p}}(\mathrm{V})=0.0465 \log \nu-$
0.6545 (Fig. 4B). For the irreversible process, $E_{\mathrm{p}}$ can be described by Tafel eqn (2),

$$
E_{\mathrm{pa}}=\left\{2.303 R T /\left[n(1-\alpha) n_{\alpha} F\right]\right\} \log \nu+K
$$

where $\alpha$ is the charge transfer coefficient, $n_{\alpha}$ is the number of electrons involved in the rate-determining step, $R$ is the 
universal gas constant $\left(8.314 \mathrm{~J} \mathrm{~K}^{-1} \mathrm{~mol}^{-1}\right), T$ is the temperature $(298 \mathrm{~K})$, and $F$ is the Faraday constant $\left(96485 \mathrm{C} \mathrm{mol}^{-1}\right.$ ). From the Tafel slope, $\alpha$ value has been calculated to be $0.45 \pm 0.03$.

\subsection{Effect of $\mathbf{p H}$}

Next, the influence of buffer $\mathrm{pH}$ on the electrochemical response of ER-GONRs/SPCE towards NIM was investigated. As the $\mathrm{pH}$ of supporting electrolyte changes, the peak current and potential of NIM reduction peak is also changed. The reduction peak current increases as the $\mathrm{pH}$ increases from 4.0 to 7.0 and reached maxima at $\mathrm{pH} 7.0$ (Fig. 4C). Afterwards, the current follows decreasing trend and hence the electrode performed poorly at basic $\mathrm{pH}$. Thus, the reduction of NIM is more favorable at $\mathrm{pH} 7.0$ and hence we have chosen $\mathrm{pH} 7.0$ as optimum $\mathrm{pH}$ for all the electrochemical studies performed in this work. The plot between different $\mathrm{pH}$ and peak potential exhibits good linearity with slope of 57.5 $( \pm 0.85) \mathrm{mV} \mathrm{pH}^{-1}$ (Fig. 4D). This slope value is close to the theoretical standard value of $-59 \mathrm{mV} \mathrm{pH}^{-1}$ for a reversible reaction involving equal numbers of protons and electrons and this result is consistent with the mechanism described in Scheme 1.

\subsection{Electroanalysis and determination of nimesulide}

Fig. 5A displays the amperometric $i-t$ curves obtained at ERGONRs/SPCE for sequential additions of NIM into PB (pH 7). The applied potential was $-0.60 \mathrm{~V}$. For each addition, a sharp increase in the amperometric current is observed and the response current reached 95\% steady-state current within $5 \mathrm{~s}$ of NIM injection. The concentration dependent linear plot displayed good linearity with slope of $0.3594 \mu \mathrm{A} \mu \mathrm{M}^{-1}$ (inset to Fig. 5A). The working concentration range was linear between $10 \mathrm{nM}$ and $1500 \mu \mathrm{M}$. The sensitivity is $1.20 \mu \mathrm{A} \mu \mathrm{M}^{-1} \mathrm{~cm}^{-2}$. The limit of detection (LOD) is calculated to be $3.50 \pm 1.57 \mathrm{nM}$ which was calculated using the formula, LOD $=3 s_{\mathrm{b}} / S$ where, $s_{\mathrm{b}}$ is the standard deviation of ten blank measurements and $S$ is the sensitivity. The performance of the sensor in terms of detection limit, working linear range, method of detection was compared with previously reported modified electrodes for NIM detection (Table 1). From the Table 1, we inferred that the overall analytical performance of ER-GONRs/SPCE is significantly better than the previously reported electrodes for NIM sensing. Notably, very low LOD $(3.50 \pm 1.57 \mathrm{nM})$ is achieved at
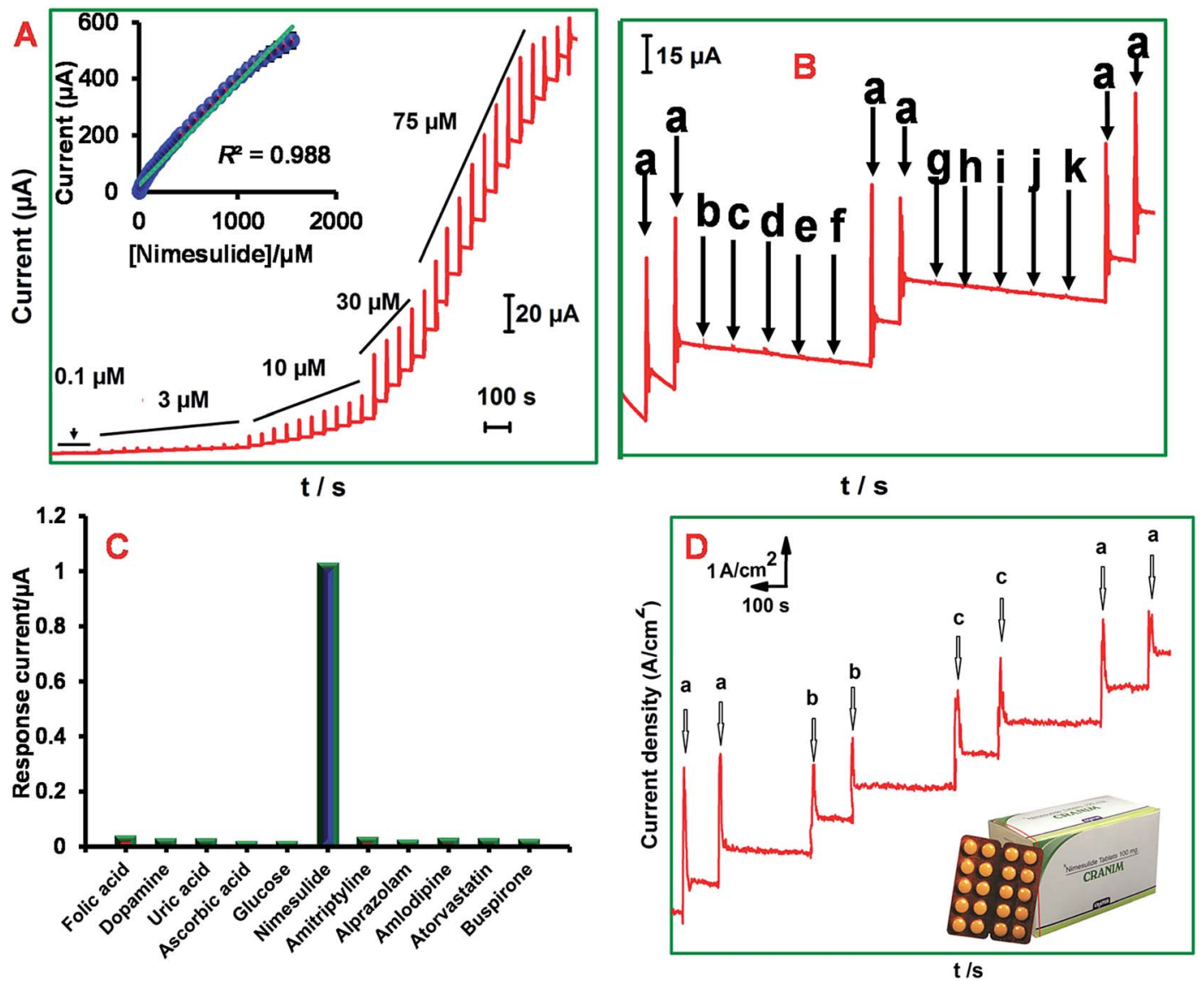

Fig. 5 (A) Amperometric response of ER-GONRs film modified electrode towards each sequential additions of NIM into PB (pH 7.0). Inset: calibration plot: [nimesulide] $/ \mu \mathrm{M}$ vs. current $(\mu \mathrm{A})$. The electrode working potential $=-0.60 \mathrm{~V}(\mathrm{vs}$. Ag/AgCl). (B) Selectivity study: amperometric response of ER-GONRs modified electrode towards $1 \mu \mathrm{M}$ of NIM (a), dopamine (b), uric acid (c), ascorbic acid (d), glucose (e), folic acid (f), amitriptyline (g), alprazolam (h), amlodipine (i), atorvastatin (j) and buspirone hydrochloride (k) into continuously stirred PB (pH 7.0). The electrode working potential $=-0.60 \mathrm{~V}$ (vs. Ag/AgCl). (C) Bar graph for selectivity study, (D) real sample analysis: amperometric responses of ER-GONRs/ SPCE for lab NIM sample (a), drug sample (NIM tablet) (b) and urine sample (c). The electrode working potential $=-0.60 \mathrm{~V}(\mathrm{vs}$. Ag/AgCl) at hydrodynamic condition. 
Table 1 Comparison of electroanalytical parameters for the determination of nimesulide at ER-GONRs/SPCE with reported works

\begin{tabular}{|c|c|c|c|c|c|}
\hline Electrode & Linear range/M & LOD & $\begin{array}{l}\text { Supporting electrolyte, } \\
\text { type of NIM catalysis }\end{array}$ & Methods & Ref. \\
\hline Cysteic acid/MWCNTs & $1.0 \times 10^{-7}$ to $1.0 \times 10^{-5}$ & $50 \mathrm{nM}$ & $0.05 \mathrm{M} \mathrm{H}_{2} \mathrm{SO}_{4}$, oxidation & $\mathrm{DPV}^{b}$ & 14 \\
\hline $\begin{array}{l}\mathrm{Fe}_{3} \mathrm{O}_{4} \text { magnetic } \\
\text { nanoparticles/GCE }\end{array}$ & $2.6 \times 10^{-6}$ to $1.0 \times 10^{-4}$ & $130 \mathrm{nM}$ & $\begin{array}{l}\text { Acetate buffer }(\mathrm{pH}=5.0) \\
\text { reduction }\end{array}$ & DPV & 12 \\
\hline Carbon paste electrode & $0.5 \times 10^{-6}$ to $10.0 \times 10^{-6}$ & $8.6 \mathrm{nM}$ & pH 7, oxidation & DPV & 32 \\
\hline $\begin{array}{l}5 \% \text { barium-doped zinc oxide } \\
\text { nanoparticle }\end{array}$ & $1.0 \times 10^{-7}$ to $1.0 \times 10^{-5}$ & $1.794 \mathrm{nM}$ & $\mathrm{PB}(\mathrm{pH} 7)$, oxidation & DPV & 11 \\
\hline $\mathrm{TiO}_{2}$ nanoparticles/GCE & $1.0 \times 10^{-7}$ to $4.0 \times 10^{-5}$ & $3.37 \mathrm{nM}$ & $\begin{array}{l}\text { Aqueous acid solution ( } \mathrm{pH} 2 \text { ), } \\
\text { oxidation }\end{array}$ & DPV & 4 \\
\hline ER-GONRs/SPCE & $1.0 \times 10^{-8}$ to $1.50 \times 10^{-3}$ & $3.50( \pm 1.57) \mathrm{nM}$ & $\mathrm{PB}(\mathrm{pH}$ 7), reduction & Amperometry & This \\
\hline
\end{tabular}

ER-GONRs/SPCE which surpassed the LOD of previously reported MWCNTs based modified electrodes, such as MWCNTs/ GCE $(\mathrm{LOD}=160 \mathrm{nM})$ and cysteic acid/MCWNTs $(\mathrm{LOD}=50 \mathrm{nM})$ based electrodes. The significantly improved sensor performance of ER-GONRs clearly indicating that ER-GONRs based modified electrode can be better transducer for the development of robust sensor for NIM assay with amplified current signal. Commonly, the reduced products of NIM are adsorbed at the electrode surface causing electrode fouling which leads to the poor sensitivity. Here, we adopt hydrodynamic conditions, in which the oxidized products are thrown away consistently and this helps resists the electrode fouling and helps the analyte to disuse easily to the electrode surface which is consistent with previous report. ${ }^{31}$

\subsection{Selectivity, reproducibility and stability}

Selectivity of the ER-GONRs/SPCE towards NIM detection was investigated in presence of possible interferents such as, biological and drugs that have electrochemical activities. The amperometric sensor responses for $1 \mu \mathrm{M}$ of NIM (a), dopamine (b), uric acid (c), ascorbic acid (d), glucose (e), folic acid (f), amitriptyline (g), alprazolam (h), amlodipine (i), atorvastatin (j) and buspirone hydrochloride $(\mathrm{k})$ are recorded at constant interval of time and given as Fig. 5B. The corresponding bar graph is given as Fig. 5C. As shown in figure, the modified electrode delivered excellent current response to each addition of NIM, however, negligible responses were observed for all the other compounds. Finally, aliquots of NIM were spiked into the aforementioned interferents co-existed electrolyte solution in order to investigate the matrix effects of those latent interferents. Nevertheless, the electrode produced sensitive signals for NIM with similar current intensities irrespective of the coexisted interferents which indicating that the ER-GONRs/SPCE has good level of selectivity to recognize and detect NIM.

In order to determine storage stability of the ER-GONRs/ SPCE, its electrocatalytic response to $2 \mu \mathrm{M}$ NIM was monitored every day. During two weeks storage period, $92.1 \%$ of initial cathodic response current was retained indicating good storage stability. Next, reproducibility of the modified electrode was evaluated by monitoring sensor performances of five electrodes in PB (pH 7) containing $2 \mu \mathrm{M}$ NIM. The RSD of five independent measurements was calculated to be $3.30 \%$ which indicating that the sensor results of ER-GONRs/SPCE can be reproducible with minimal error.

\subsection{Real sample analysis}

All the excellent sensor features of ER-GONRs/SPCE, such as ultra-sensitivity, good selectivity, excellent reproducibility and appreciable stability are revealing that this electrode can be suitable for real-time analysis of pharmaceutical and biological samples. The preparation of real samples is given in the Experimental section. The amounts of NIM present in tablet and urine samples were tested using our ER-GONRs/SPCE electrode. Amperometric experiments were conducted using ER-GONRs/ SPCE, while lab and real samples are spiked into the supporting electrolyte at appropriate interval as shown in Fig. 5D. As expected the lab samples have shown prompt and sensitive signals. Interestingly the corresponding concentrations of real samples (drug and urine) have shown sensitive signals which are consistent with the current values of lab samples. The recoveries are calculated and found to be in the acceptable range of 96.898.6\% (Table 2). Thus, the modified electrode has excellent practical feasibility to be used in real-time applications.

Table 2 Determination of NIM in pharmaceutical and biological samples

\begin{tabular}{llcll}
\hline Real samples & Added/nM & Found/nM & Recovery/\% & $\operatorname{RSD}^{a} / \%$ \\
\hline \multirow{2}{*}{ NIM drug } & 100 & 96.8 & 96.8 & 4.52 \\
\multirow{2}{*}{ Urine } & 200 & 195.2 & 97.6 & 4.91 \\
& 100 & 98.6 & 98.6 & 4.33 \\
& 200 & 194.0 & 97 & 4.70
\end{tabular}

${ }^{a}$ Related standard deviation (RSD) of 3 independent experiments. 


\section{Conclusions}

In summary, an ultra-sensitive, reproducible, stable and realtime applicable modified electrode, ER-GONRs/SPCE was developed for the determination of NIM. The ER-GONRs was characterized by TEM, EDX, XRD, Raman, FT-IR, and impedance methods. The modified electrode delivered excellent electrocatalytic ability towards NIM reduction. The developed amperometric sensor has excellent sensor performance and exhibited wide linear range $\left(1.0 \times 10^{-8}\right.$ to $\left.1.50 \times 10^{-3}\right)$, low detection limit $(3.50 \mathrm{nM})$, high sensitivity $\left(1.20 \mu \mathrm{A} \mu \mathrm{M}^{-1} \mathrm{~cm}^{-2}\right)$ and fast response $(<5 \mathrm{~s})$. The studies revealed that the ERGONRs have significantly improved sensor performance in comparison with pristine MWCNTs and other previously developed chemical modifiers. The assay demonstrated in drug and urine samples revealed the good practical feasibility of the ER-GONRs/SPCE in drug and clinical analysis.

\section{Acknowledgements}

This work was supported by the Ministry of Science and Technology (MOST), Taiwan (ROC) and MacKay Memorial Hospital, NTUT-MMH-106-06.

\section{References}

1 P. F. Pereira, M. C. Marra, A. B. Lima, W. T. P. dos Santos, R. A. A. Munoz and E. M. Richter, Diamond Relat. Mater., 2013, 39, 41.

2 I. S. da Silva, B. Capovilla, K. H. G. Freitas and L. Angnes, Anal. Methods, 2013, 5, 3546.

3 I. Bjarnason and B. Thjodleifsson, Rheumatology, 1999, 38, 24.

4 S. D. Bukkitgar, N. P. Shetti, R. M. Kulkarni, S. B. Halbhavi, M. Wasim, M. Mylar, P. S. Durgi and S. S. Chirmure, J. Electroanal. Chem., 2016, 778, 103.

5 M. Carrasco-Portugal, V. Granados-Soto, G. Camacho-Vieyra, J. Pérez-Urizar and F. J. Flores-Murrieta, J. Liq. Chromatogr. Relat. Technol., 2000, 23, 2237.

6 X. Sun, K.-L. Xue, X.-Y. Jiao, Q. Chen, L. Xu, H. Zheng and Y.-F. Ding, J. Chromatogr. B: Anal. Technol. Biomed. Life Sci., 2016, 1027, 139.

7 J. Wang, Analytical electrochemistry, John Wiley \& Sons, 2006. 8 A. J. Bard and L. R. Faulkner, Electrochemical methods: fundamentals and applications, Wiley, New York, 1980.

9 A. Álvarez-Lueje, P. Vasquez, L. Núñez-Vergara and J. Squella, Electroanalysis, 1997, 9, 1209.
10 S. J. Malode and S. T. Nandibewoor, Asian J. Pharm. Clin. Res., 2013, 6, 71.

11 S. D. Bukkitgar, N. P. Shetti, R. M. Kulkarni and M. R. Doddamani, J. Electroanal. Chem., 2016, 762, 37.

12 J. Zhang, X. Tan, D. Zhao, S. Tan, L. Liu, L. Wang and Z. Huang, Chem. Res. Chin. Univ., 2011, 27, 566.

13 J. Zhang, X. Tan, D. Zhao, S. Tan, Z. Huang, Y. Mi and Z. Huang, Electrochim. Acta, 2010, 55, 2522.

14 C. Wang, X. Shao, Q. Liu, Q. Qu, G. Yang and X. Hu, J. Pharm. Biomed. Anal., 2006, 42, 237.

15 F. Valentini, D. Romanazzo, M. Carbone and G. Palleschi, Electroanalysis, 2012, 24, 872.

16 L. Li, A.-R. O. Raji, H. Fei, Y. Yang, E. L. Samuel and J. M. Tour, ACS Appl. Mater. Interfaces, 2013, 5, 6622.

17 R. Zhang, C.-L. Sun, Y.-J. Lu and W. Chen, Anal. Chem., 2015, 87, 12262.

18 H. Jin, H. Huang, Y. He, X. Feng, S. Wang, L. Dai and J. Wang, J. Am. Chem. Soc., 2015, 137, 7588.

19 C. Wang, H. Li, J. Zhao, Y. Zhu, W. Z. Yuan and Y. Zhang, Int. J. Hydrogen Energy, 2013, 38, 13230.

20 L. Shang, F. Zhao and B. Zeng, Electrochim. Acta, 2015, 168, 330.

21 S. Wu, X. Lan, F. Huang, Z. Luo, H. Ju, C. Meng and C. Duan, Biosens. Bioelectron., 2012, 32, 293.

22 A. Zehtab Yazdi, E. P. L. Roberts and U. Sundararaj, Carbon, 2016, 100, 99.

23 C.-L. Sun, C.-T. Chang, H.-H. Lee, J. Zhou, J. Wang, T.-K. Sham and W.-F. Pong, ACS Nano, 2011, 5, 7788.

24 Y. Yi, G. Zhu, X. Wu and K. Wang, Biosens. Bioelectron., 2016, $77,353$.

25 A. L. Higginbotham, D. V. Kosynkin, A. Sinitskii, Z. Sun and J. M. Tour, ACS Nano, 2010, 4, 2059.

26 A. Martín, J. Hernández-Ferrer, L. Vázquez, M.-T. Martínez and A. Escarpa, RSC Adv., 2014, 4, 132.

27 M. Thiruppathi, N. Thiyagarajan, M. Gopinathan and J.-M. Zen, Electrochem. Commun., 2016, 69, 15.

28 V. Mani, B. Devadas and S.-M. Chen, Biosens. Bioelectron., 2013, 41, 309.

29 C. H. A. Wong and M. Pumera, J. Mater. Chem. C, 2014, 2, 856.

30 C. H. A. Wong, C. K. Chua, B. Khezri, R. D. Webster and M. Pumera, Angew. Chem., Int. Ed., 2013, 125, 8847.

31 B. Unnikrishnan, V. Mani and S.-M. Chen, Sens. Actuators, B, 2012, 173, 274.

32 S. J. Malode and S. T. Nandibewoor, J. Phys. Chem., 2013, 227, 73. 